\title{
Comparative Evaluation of Total Phenolics, Total Flavonoids Content and Antiradical Activity in Six Selected Species of Family Rosaceae Using Spectroscopic Method
}

\author{
Kiran Zahid ${ }^{1 *}$, Maqsood Ahmed ${ }^{2}$ and Farah Khan ${ }^{3}$ \\ 1,3 Department of Botany, Lahore College for Women University, Pakistan \\ ${ }^{2}$ Lahore Pharmacy College and Lahore Medical and Dental College, Pakistan \\ *Corresponding author: Kiran Zahid, Department of Botany, Lahore College for Women University, Lahore, Pakistan. \\ To Cite This Article: Kiran Zahid. Comparative Evaluation of Total Phenolics, Total Flavonoids Content and Antiradical Activity in Six Selected \\ Species of Family Rosaceae Using Spectroscopic Method. Am J Biomed Sci \& Res. 2019 - 3(4). AJBSR.MS.ID.000693. \\ DOI: $10.34297 / A J B S R .2019 .03 .000693$
}

Received: June 11, 2019 | Published: June 25, 2019

\begin{abstract}
The current study aims to investigate the total phenolics, total flavonoids content in addition to radical scavenging activity in six selected species of Family Rosaceae. Double Maceration Technique was adopted for extraction process. Results indicated the presence of phytochemicals in three species i.e. Pyrus communis, Prunus armeniaca \& Malus pumila while absence of alkaloids and terpenoids was reflected in other three species i.e. Prunus persica, Prunus domestica \& Fragaria vesca. Maximum Phenolic and flavonoids content was found in Fragaria vesca (leaf) using $70 \%$ ethanol as a solvent. All the tested plant species exhibited DPPH anti-radical properties however Prunus domestica (Leaf) $96.06 \pm 0.57$ possessed maximum antioxidant potential with IC50 value of $0.236 \pm 0.001 \mathrm{mg} / \mathrm{mL}$ using Graph pad Prism 5.04 software. Ethanolic extracts gave preferable results and have the potential in extracting phytochemicals; these phytochemicals played tremendous role in treating multiple disorders including anticancer, antidiabetic and neurological disorders like Alzheimer or Dementia.
\end{abstract}

Keywords: Polyphenolics, Flavonoids, Half maximal inhibitory concentration (IC50), Phytomedicine

\section{Introduction}

Polyphenolics like phenolics and flavonoids exhibit diversify range of biological activities. These Phyto-compounds play a defensive role against antimicrobial diseases [1]. Fruits are the enrich source of polyphenolic compounds and utilization of fruits have showed a significant role in curing countless human disorders [2]. All the selected species of Family Rosaceae possessed therapeutic importance i.e. Pyrus communis aerial and flowering parts are a source of traditional medicine as they exhibit anti-inflammatory properties. [3] Prunus armeniaca possessed wound healing properties in normal rats, Prunus armeniaca is the enrich source of natural antioxidant and play an important role against cardiovascular diseases, anti-microbial as well as skin related issues [4], Malus pumila possessed polyphenols that have beneficial effects on health, such as exhibiting antioxidant activity, antibacterial, anti-inflammatory, anti-radiation, antitumor and anti-obesity effect [5,6], Prunus persica leaf and fruit exhibited anti-tumor, anti-allergic, anti-inflammatory, anti-oxidant activities [7], Prunus domestica possessed various phenolic acids and flavonoids which allow their use as nutraceuticals in current market due to their preventive and therapeutic nature [8], The berries, leaves, and roots of Fragaria vesca have all been used as a folk medicine for diarrhea and the stalks for wounds, for cardiovascular diseases like cancer and heart related disorders [9].

Natural antioxidants gain immense importance as they impose less or no side effects in comparison to Synthetic antioxidants. Antioxidants played a significant role in scavenging free radicals as free radicals lead to serious health issues like neurological disorders, Cancer and so on. Natural antioxidants are of great concern for the researchers of this field as they have shown profound results in comparison to synthetic antioxidants [10]. The present study illustrates the qualitative phytochemical screening and quantitative profiling of total phenolics, flavonoids content and anti-radical 
potential of selected six species of Family Rosaceae. Ethanol gave preferable results in comparison to methanol extracts. A significant correlation exhibited between all the parameters which impart a specific and important role in many biological activities. Hence this research provides base line for the use of these plant species in different biological activities which include anti-cancer, antidiabetic and neurological disorders etc.

\section{Experimental Section}

\section{Plant Material}

The plant parts (Leaves/Bark/Fruit) of all the selected six species of Family Rosaceae were collected from Jinnah Garden, Lahore in the months of Feb-May (2016), authenticated and identified by Dr. Mir Ajab Ali Khan, Professor Department of Biological Sciences, Quaid-e-Azam University, Islamabad, deposited in the Prem Madan Herbarium of Lahore College for Women
University, Lahore (Pyrus communis Voucher No: LCWU-15-123, Prunus armeniaca Voucher No: LCWU-15-125, Malus pumila Voucher No: LCWU-15-116, Prunus persica Voucher No: LCWU-15124, Prunus domestica Voucher No: LCWU-15-99, Fragaria vesca Voucher No: LCWU-15-127).

\section{Extracts Preparation}

Leaves/ fruit/ bark of selected six species undergo double maceration protocol for the extraction of plant extracts at $21^{\circ} \mathrm{C}$ via two polar solvents i.e. methanol and ethanol [11].

\section{Phytochemical Screening}

Phytochemical screening was performed for alkaloids, phenolic acids, flavonoids, terpenoids and tannins. The color intensity or the precipitate formation was used as analytical responses to these tests (Table 1) [12-19].

Table 1: Phytochemical Screening of six selected species of Family Rosaceae.

\begin{tabular}{|c|c|c|c|c|c|}
\hline \multirow{2}{*}{ Plant species } & \multicolumn{5}{|c|}{ Results } \\
\hline & Terpenoids & Alkaloids & Flavonoids & Phenolics & Tannins \\
\hline Pyrus communis L. (Leaf) & ++ & ++ & ++ & ++ & ++ \\
\hline Prunus armeniaca L. (Fruit) & ++ & ++ & ++ & ++ & ++ \\
\hline Malus pumila Auct. (Leaf) & ++ & ++ & ++ & ++ & ++ \\
\hline Prunus persica L. (Leaf) & -- & -- & ++ & ++ & ++ \\
\hline Prunus domestica L. (Bark) & ++ & -- & ++ & ++ & ++ \\
\hline Prunus domestica L. (Leaf) & ++ & -- & ++ & ++ & ++ \\
\hline Fragaria vesca L. (Leaf) & ++ & -- & ++ & ++ & ++ \\
\hline
\end{tabular}

Quantitative Detection of Total Phenolics \& Flavonoids Content

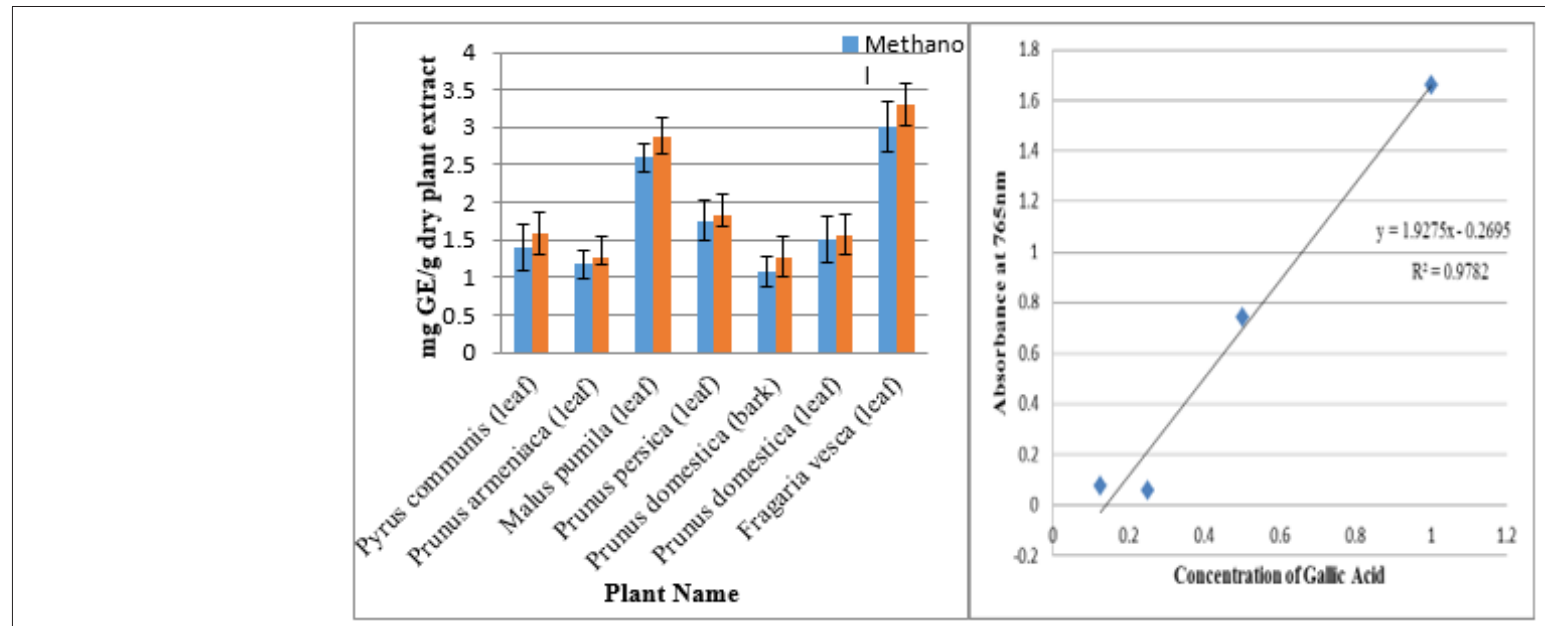

Figure 1a: Calibration curve for Gallic Acid.

Figure 1b: Total phenolic content (mg Gallic acid/g dry plant extract).

Folin-Ciocalteu (FC) reagent was used to assess total phenolic content in the test sample, phenolics presence in the test sample resulted in formation of blue complex (phosphotungstatephosphomolybdate) using gallic acid as a standard. Gallic acid calibration curve provides as a baseline for the determination of TPC content $[20,21]$ (Figure $1 \mathrm{a} \& 1 \mathrm{~b}$ ). Aluminium chloride $\left(\mathrm{AlCl}_{3}\right)$ method was used for the detection of total flavonoid content (TFC), on reaction of test sample and aluminium chloride appearance of yellow color is the indication of presence of flavonoids. Quercetin calibration curve was used as a standard for the measurement of flavonoids (Figure 2a \& 2b) [22,23]. 

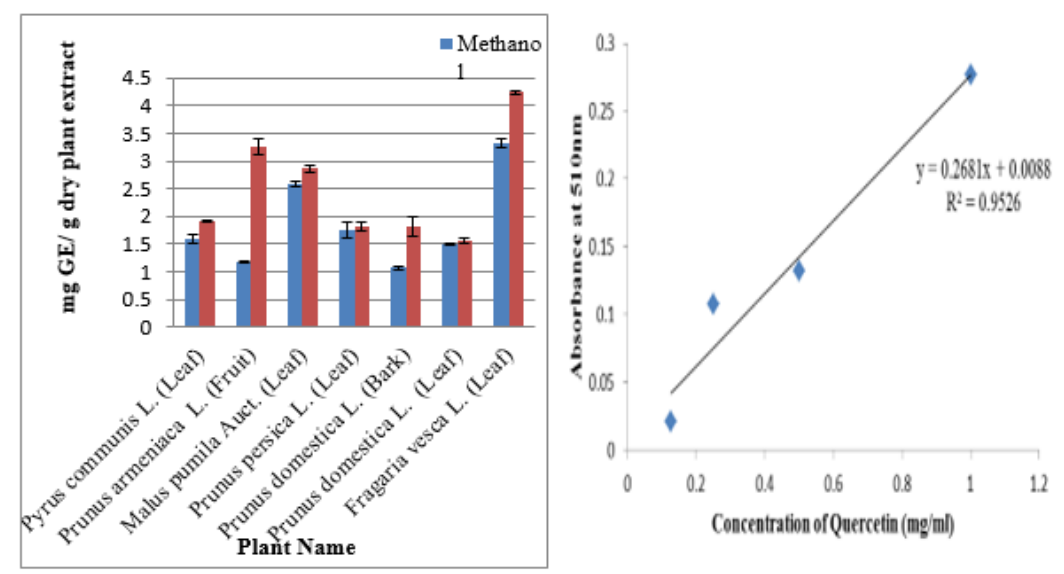

Figure 2a: TFC (mg Quercetin/ g dry plant extract).

Figure 2b: Calibration Curve of Quercetin ( $\mathrm{mg} / \mathrm{ml})$.

\section{Antioxidant Potential}

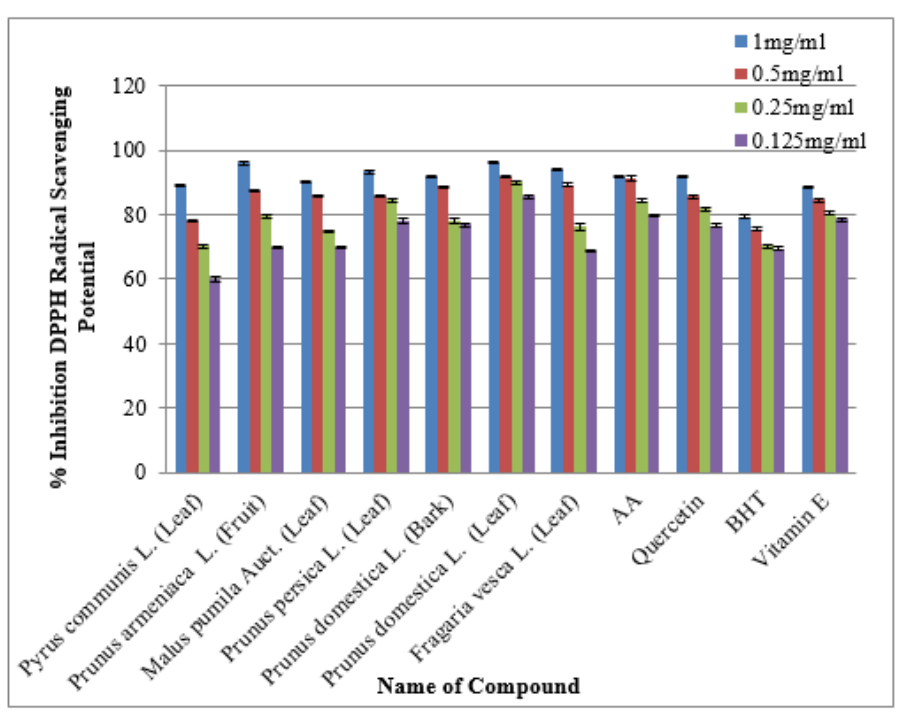

Figure 3: \% inhibition DPPH Radical Scavenging Activity using Methanol as a Solvent.

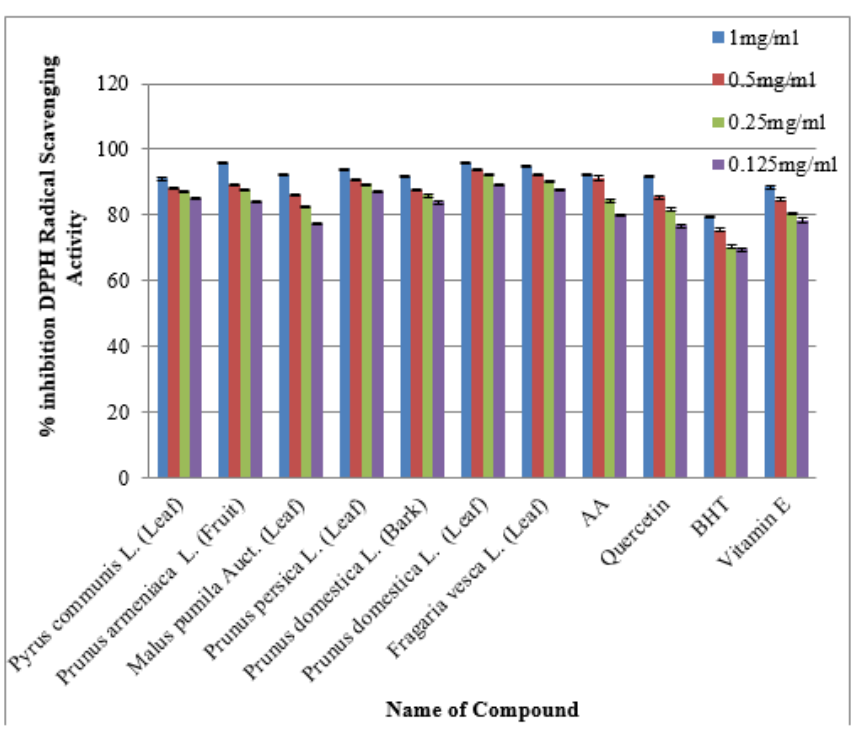

Figure 4: \% inhibition DPPH Radical Scavenging Activity of six species of Family Rosaceae using Ethanol as a Solvent 
Methanolic \& Ethanolic Leaves/Bark/Fruit extract of selected species of Family Rosaceae was estimated for radical scavenging activity by means of DPPH assay. Tested samples were assessed at four different concentrations i.e. $0.125,0.25,0.5$ and $1 \mathrm{mg} /$ $\mathrm{mg} / \mathrm{mL}$. Add $50 \mu \mathrm{L}$ plant extracts in methanol/ethanol solution of DPPH ( $5 \mathrm{ml}$ of $0.05 \mathrm{mM}$ ) leave it for half hour then absorbance was measured at $517 \mathrm{~nm}$. Conversion of deep-violet color to light yellow is the clear indication of the presence of antioxidants. Results were compared with the positive control used in the test (Figure $3 \& 4$ ) Half minimal concentration was assessed by means of Graph pad prism 5.04 software (Figure 5) [24,25].

\section{Statistical Analysis}

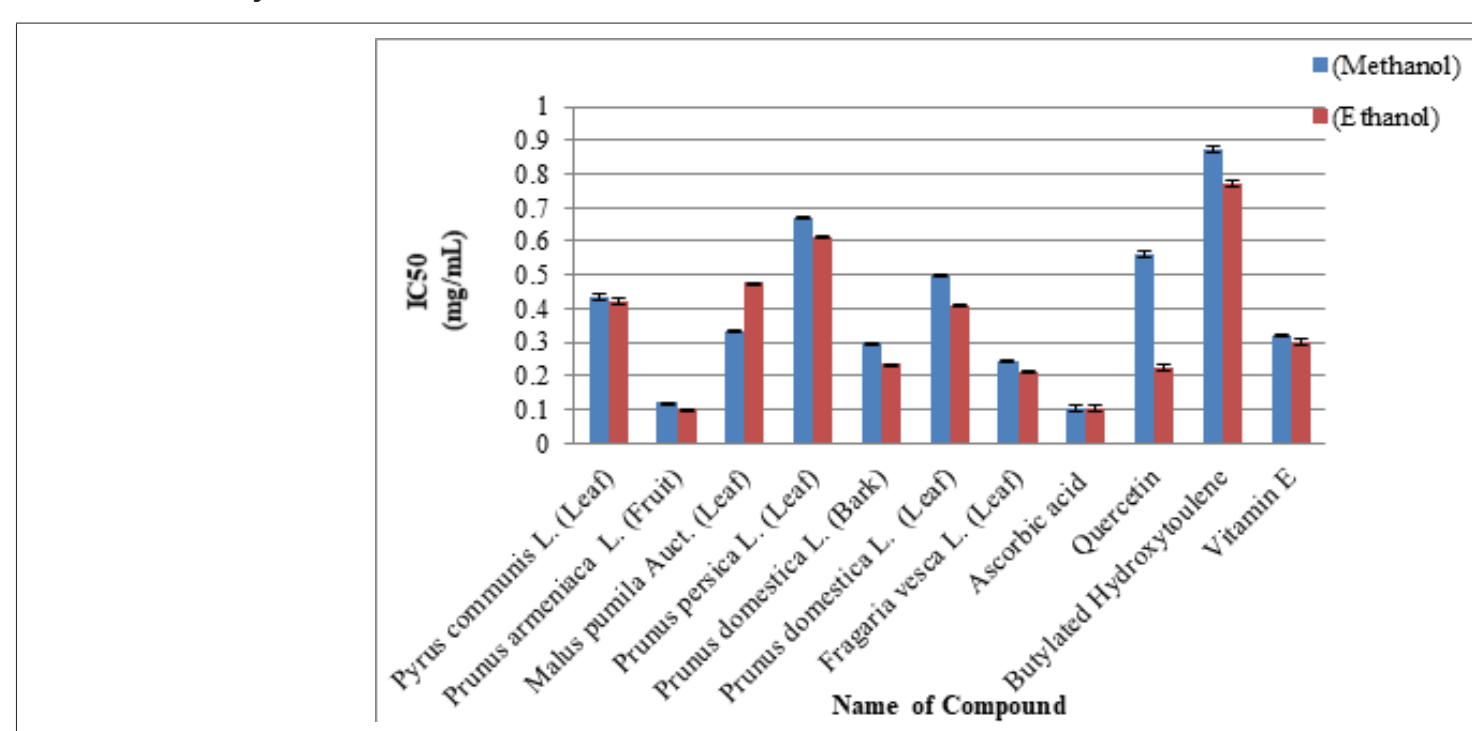

Figure 5: Half Maximal Inhibitory Concentration (IC50) of six species of Family Rosaceae along with artificial antioxidants.

Mean \pm Standard deviation of three parallel measurements were done using SPSS software, Microsoft Excel and Graph pad prism Software 5.04 [26].

\section{Results and Discussion}

The results were gathered in Table 1. It is noteworthy that the presence of flavonoids, phenolics and tannins in all six species of Family Rosaceae, terpenoids presence was indicated in all the five species which absence of terpenoids was reflected in $P$. persica. However, absence of alkaloids was observed in Prunus persica, Prunus domestica, Fragaria vesca while other three species Pyrus communis, Prunus armeniaca, Malus pumila showed the presence of alkaloids (Table 1).

Six selected plant species of family Rosaceae was assessed for total phenolic content (TPC). Among tested samples Fragaria vesca (Leaf) showed Maximum TPC content i.e. 3.314 $\pm 0.28 \mathrm{mg}$ GE/ g dry plant extract while minimum phenolic content was reflected in Prunus domestica (bark) $1.0699 \pm 0.2 \mathrm{mg} \mathrm{GE} /$ dry plant extract using $70 \%$ ethanol as solvent. Ethanol proved to be an excellent solvent in extracting phenolic acids at both inter and intra cellular level [27]. Similar outcomes were obtained by the work of different researchers [28,30]. Results were given in Figure 1. Most diversified groups of phytochemicals found in all plant organs are termed as Phenolics [31]. Phenolic compounds found in plants have a diversified range of functions including anti-inflammatory, antiradical, antitumor, antidepressant activities etc. and so on [32]. Variation in the Phenolics content was observed based on solvent used [33].
Results showed that $70 \%$ ethanol was used for extracting maximum flavonoids content by Fragaria vesca i.e. $(4.84 \pm 0.17$ $\mathrm{mg}$ QE/g of dry extract) while $2.952 \pm 0.13 \mathrm{mg}$ of QE equivalents (GAE)/g dry weight flavonoid content was achieved with methanol. Role of flavonoids was well known in the medical field as it prevents from antimicrobial, cancerous and hepatoprotective problems [34]. Figure $2 a \& 2 b$ illustrate the following results.

DPPH assay was used for estimating radical scavenging activities in six selected species of Family Rosaceae and Moraceae. All the selected species of Family Rosaceae and Moraceae showed strong antiradical capabilities in comparison to the positive drugs used i.e. Ascorbic Acid, Quercetin, Butylated Hydroxytoulene and Vitamin E (Figure 1\&2): Antioxidant potential was found to be best in ethanol rather than methanol (Figure 3\&4) and was in the following order: Prunus domestica (Leaf) $\geq$ Prunus armeniaca (Fruit) $\geq$ Fragaria vesca (Leaf) $\geq$ Prunus persica (Leaf) $\geq$ Pyrus communis (Leaf) $\geq$ Prunus domestica (Bark) 96.13 $\pm 0.21 \geq 96.06 \pm 0.27 \geq 94.92 \pm 0.21 \geq$ $94.22 \pm 0.04 \geq 91.70 \pm 0.40 \geq 91.70 \pm 0.40$.

Results obtained from the current research work strongly correlate with the work of different researchers observed that presence of secondary metabolites gave $P$. acerifolium leaves strong radical scavenging abilities. Antioxidant rich plants have shown significant potential in the curing of multiple disorders like antitumor, proapoptotic and antiangiogenic effects [35]. IC50 value was also calculated with the help of Graph pad Prism 5.04 software (Figure 5). 


\section{Conclusion}

Hence, conclude that all the six species of Family Rosaceae are enriched source of phytochemicals like phenolics, terpenoids, alkaloids, tannins and flavonoids and the radical scavenging properties of all the six species is due to the phytochemicals present in them. Results showed there is a strong correlation between phytochemicals and anti-radical properties of plant samples under investigation. Maximum Phenolic and Flavonoid contents were found in Fragaria vesca L. using 70\% ethanol as solvent. This research is of great significance as all species acted as potent antioxidants and thereby lead as a baseline for the treatment of multiple disorders like anti-cancer, antidiabetic properties, neurological disorders like Alzheimer or Dementia.

\section{Acknowledgements}

Special thanks to Dr. Mir Ajab Ali Khan, Professor Department of Biological Sciences, Quaid-e-Azam University, Islamabad for identification of plants.

\section{References}

1. Babbar N, Oberoi HS, Sandhu SK, Bhargav VK (2014) Influence of different solvents in extraction of phenolic compounds from vegetable residues and their evaluation as natural sources of antioxidants. J Food Sci Technol 51(10): 2568-2575.

2. Manach C, Scalbert A, Morand C, Remesy C, Jimenez L (2004) Polyphenols: Food sources and bioavailability. Am J Clin Nutr 79(5): 727-747.

3. Velmurugan C, Bhargava A (2014) Total phenolic, flavonoids and tannin content of various extracts from Pyrus communis fruit. IJPAR 3(4): 384390.

4. Yigit D, Yigit N, Mavi A (2009) Antioxidant and antimicrobial activities of bitter and sweet apricot (Prunus armeniaca L) kernels. Braz J Med Biol Res 42(4): 346-352.

5. Hyson DA (2011) A comprehensive review of apples and apple components and their relationship to human health. Adv Nutr 2(5): 408-420.

6. Shao J W, Dai YC, Xue JP, Wang JC, Lin FP, et al. (2011) In vitro and in vivo anticancer activity evaluation of ursolic acid derivatives. Eur J Med Chem 46(7): 2652-2661.

7. Kashyap D, Kumar S, Dhatwalia V (2015) Review on phytochemical and pharmacological properties of Prunus persica Linn. Asia-Pac J Chem Eng 2: 5-11.

8. Fan J, Ding X, Gu W (2007) Radical-scavenging proanthocyanins from sea buckthorn seed. Food Chem 102(1): 168-177.

9. Bombarely A, Merchante C, Csukasi F, Cruz-Rus E, Caballero JL, et al. (2010) Generation and analysis of ESTs from strawberry (Fragaria $x$ ananassa) fruits and evaluation of their utility in genetic and molecular studies. BMC Genomics 11(17): 503.

10. Pramod J, Singh S, Singh J (2013) Role of Free Radicals and Antioxidants in Human Health and Disease. IJCRR 5: 14-22.

11. Bandar H, Hijazi A, Rammal H, Hachem A, Saad Z, et al. (2013) Techniques for the extraction of bioactive compounds from Lebanese Urtica Dioica. AJPCT 1(6): 507-513.

12. Salehi-Surmaghi MH, Aynehchi Y, Amin GH, Mahhmoodi Z (1992) Survey of Iranian plants for saponins, alkaloids, flavonoids and tannins IV DARU. 2:1-11.
13. Abdullahi MN, Ilyas N, Ibrahim H (2013) Evaluation of phytochemical screening and analgesic activity of aqueous extract of the leaves of Microtrichia perotitii dc (Asteraceae) in mice using hotplate method. Med Plant Res 3(5): 37-43.

14. Joshi A, Bhobe M, Saatarkar A (2013) Phytochemical investigation of the roots of Grewia microcos Linn. J Chem and Pharm Res 5(7): 80-87.

15. Harbor JB (1973) Photochemical methods-A Guide to Modern Techniques of Plant Analysis Chapman and Hall. London pp. 49-188.

16. Tiwari P, Kumar B, Kaur M, Kaur G, Kaur H (2011) Phytochemical Screening and Extraction: A Review. Internationale Pharmaceutica Sciencia 1: 98-106.

17. Umesh, BT, Hermalatha S, Anuj M (2010) Pharmacognostic and phytochemical investigation on root of Cadaba farinosa Forsk. IJPBS 1: $1-13$.

18. Indumathi C, Durgadevi G, Nithyavani S, Gayathri PK (2014) Estimation of terpenoid content and its antimicrobial property in Enicostemma litorrale. Int J Chemtech Res 6: 4264-4267.

19. Kannan V, Mohamed Fahad S, Siva Arumugam, CD, Vinoth Kumar RBN (2015) Phytochemical screening of Bauhinia purpurea important medical plant. IRJP 6: 2230-8407.

20. Cliffe S, Fawer MS, Maier G, Takata K, Ritter G (1994) Enzyme assays for the phenolic content of natural juices. J Agric Food Chem 42:1824-1828.

21. Chlopicka J, Pasko P, Gorinstein S, Jedryas A, Zagrodzki P (2012) Total phenolic and total flavonoid content, antioxidant activity and sensory evaluation of pseudocereal breads. LWT-Food Sci and Tech 46: 548-555.

22. Dewanto VX, Wu K, Adom K, Liu RH (2002) Thermal processing enhances the nutritional value of tomatoes by increasing total antioxidant activity. J Agric Food Chem 50(10): 3010-3014.

23. Stankovic MS (2011) Total phenolic content, flavonoid concentration and antioxidant activity of Marrubium peregrinum L. extracts. Kragujevac J Sc 33: 63-72.

24. Lee SK, Mbwambo ZH, Chung HS, Luyengi L, Games ECJ et al. (1998) Combinatorial Chemistry and High Throughput Screening. p.1: 35.

25. Erasto P, Moleta GB, Majinda RRT (2004) Antimicrobial and antioxidant flavonoids from the root wood of Bolusanthus speciosus. Phytochemistry 65(7): 875-880.

26. Levesque R (2007) SPSS Programming and Data Management: A Guide for SPSS and SAS users, $4^{\text {th }}$ Edn, SPSS Inc, Chicago III, US.

27. Vamanu E, Vamanu A, Nita S and Colceriu S (2011) Antioxidant and antimicrobial activities of ethanol extracts of Cynara scolymus (Cynarae folium, Asteraceae family). Trop J Pharm Res 10(6): 777-783.

28. Akond ASMGM, Khandaker, L, Hossain KG, Furuta Y (2010) Total Polyphenol, Polyphenol Oxidase, Antioxidant Activity and Color Profiles of Some Wheat Varieties from Bangladesh. Res J Agric Biol Sci 6: 186190.

29. Carlsen MH, Halvorsen BL, Holte K, Bohn SK, Dragland S, et al. (2010) The total antioxidant content of more than 3100 foods, beverages, spices, herbs and supplements used worldwide. Nutr J 3(9): 2891-2899.

30. Sannigrahi S, Parida S, Patro VJ, Mishra US, Pathak A (2010) Antioxidant and anti-inflammatory potential of Pterospermum acerifolium. IJPSR 2: 1-5.

31. Shahidi F, Chandrasekara A (2013) Millet grain phenolics and their role in disease risk reduction and health promotion. J Functional Foods 5: 570-581.

32. Ghasemzadeh A, Jaafar HZE, Rahmat A (2011) Effects of solvent type on phenolics and flavonoids content and antioxidant activities in two varieties of young ginger (Zingiber officinale Roscoe) extracts. J Med Plants Res 5(7): 1147-1154. 
33. Marmouzi I, Madani NE, Charrouf Z, Cherrah Y, Faouzi MEA (2015) Proximate analysis, fatty acids and mineral composition of processed Moroccan Chenopodium quinoa Wild and antioxidant properties according to the polarity. Phytothérapie 13(2): 110-117.

34. Kumar S, Pandey AK (2013) Chemistry and biological activities of flavonoids: an overview. Sci World J 29: 162750
35. Carocho M, Ferreira ICFR (2013) The role of phenolics compounds in the fight against cancer-a review. Anticancer Agents Med Chem 13(8): 12361258. 